\title{
Beata Beatrix: la Vita Nuova e i quadri di Dante Gabriel Rossetti
}

\author{
Veronica Pesce \\ Università di Genova \\ veronica.pesce@unige.it
}

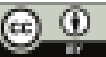

\section{Riassunto}

Il contributo intende proporre un primo percorso attraverso la rilettura della Vita Nuova in ambito preraffaellita, esaminando la raffinata edizione della Vita Nuova illustrata dai quadri di Dante Gabriel Rossetti pubblicata per Roux e Viarengo all'inizio del '9oo. L'edizione, postuma, non nasce da un preciso progetto illustrativo autoriale, pur accarezzato dal poeta-pittore fin da giovanissimo, ma mette insieme a posteriori le riproduzioni di alcune opere rossettiane a illustrazione del libello dantesco, non senza arbitrarietà. Emerge tuttavia un costante impegno dell'artista su questi soggetti che, accanto al lavoro di traduzione sulla stessa Vita Nuova e sui "poeti primitivi italiani", e insieme con la sua produzione poetica e letteraria (almeno The Blessed Damozel e Hand and Soul), dimostra una profonda riappropriazione del testo, caratterizzata al contempo da una certa fedeltà letterale non in contraddizione con una rivisitazione profonda in tutt'altro contesto storico-artistico.

Parole chiave: Dante Alighieri, Vita Nuova, Dante Gabriel Rossetti, Preraffaelliti, Beatrice.

\begin{abstract}
The aim of the paper is to propose a first path through the rereading of the Vita Nuova within the Pre-Raphaelite culture, examining the refined edition of the Vita Nuova illustrata dai quadri di Dante Gabriel Rossetti, published by Roux and Viarengo in early '9oo. This posthumous edition is not the result of a specific illustrative authorial project, although the poet-painter had aimed for this since his early age; it is rather an a posteriori and partly arbitrary collection, which puts together the reproductions of some Rossetti's illustrations from Dante's pamphlet. The artist never stopped working on these subjects, translating at the same time Dante's Vita Nuova and other Early Italian Poets, while working on his own poetic and literary production (The Blessed Damozel and Hand and Soul, at least): this demonstrates a deep repossession of the text, which is characterized by a certain literal accuracy, but also by a deep revision, according to a completely different historical and artistic context.
\end{abstract}

Keywords: Dante Alighieri, Vita Nuova (The New Life), Dante Gabriel Rossetti, PreRaphaelites, Beatrice. 
And did'st thou know indeed, when at the font Together with thy name thou gav'st me his, That also on thy son must Beatrice Decline her eyes according to her wont [...]. D. G. Rossetti, Dantis Tenebrae. In Memory of my Father

W el novembre del I848, annunciando in una lettera a Charles Lyell di aver 1 completato la traduzione della Vita Nuova, Dante Gabriel Rossetti rende conto con puntualità del suo progetto illustrativo:

[...] intending it to accompany a series of original designs which I have commenced in illustration of that work. [...] Of the designs I have completed, as yet, only three: $\mathrm{I}^{\text {st }}$ Dante overhearing the conversation of the friends of Beatrice after the death of her father: $2^{\text {nd }}$ Dante interrupted while drawing an angel in memory of Beatrice: $\& 3^{\text {rd }}$ an emblematical frontispiece. In this last, I have introduced on one side the figure of Dante and on the other that of Beatrice: while in the centre, Love is represented, holding in one hand a sun-dial, and in the other a lamp; the shadow cast by the lamp upon the dial being made to fall upon the figure nine. At the same time, Death, standing behind, is drawing from the quiver of Love an arrow wherewith to strike Beatrice. Ever since I have read the "Vita Nuova", I have always borne it in mind as a work offering admirable opportunities for pictorical illustration: a task which I am now resolved to attempt. The other subjects which I propose treating are as follows: $\mathrm{I}^{\text {st }}$ The first meeting of Dante and Beatrice (already commenced): $2^{\text {nd }}$ The second meeting: $3^{\text {rd }}$ The salutation denied: $4^{\text {th }}$ Dante treated with scorn by Beatrice at the Wedding-feast: $5^{\text {th }}$ Dante questioned by the ladies: $6^{\text {th }}$ Dante's dream: $7^{\text {th }}$ Dante requested to write by the kinsman of Beatrice: $8^{\text {th }}$ Dante perceives a lady who is observing his grief from a window: $9^{\text {th }}$ Dante's vision of the childhood of Beatrice: $\mathrm{IO}^{\text {th }}$ Dante and the pilgrims (Fredeman 2002-20IO: 76).

La preziosa lettera dimostra un preciso progetto che discende dal profondo valore della Vita Nuova per Dante Gabriel; ${ }^{2}$ il libello è letto e fors'anche tradotto pensando già alla sua restituzione pittorica e pare valere quale vera e propria summa dell'arte di Dante Gabriel: "a means of interpreting his own life and $[. .$.$] a way in to an interior world of ideal love, that became one of the$

I. Battezzato Gabriel Charles Dante Rossetti, cambierà in seguito l'ordine dei nomi, così come oggi li conosciamo: Dante Gabriel Rossetti. Il testo citato è pubblicato per la prima volta nell'edizione dei Poems (Londra, Ellis, 1870).

2. Camilletti fa osservare quanto sia sintomatica di una profonda riappropriazione la dedica ("Whatever is mine in this book is inscribed to my wife") apposta alla traduzione di The Early Italian Poets (vedi infra): “'Tutto ciò che è mio': la donna è concretizzazione di sogni, anima, l'opera stessa dell'artista/amante" (Camilletti 2005: 92). 
well-springs of his art" (Becker 2004: I8); si tratta evidentemente di una Vita Nuova già passata attraverso la temperie romantica e ora rimodellata dall'estetismo ${ }^{3}$ e dal simbolismo incipienti, senza considerare il filtro dell'interpretazione occultista e iniziatica di Gabriele Rossetti padre. ${ }^{4}$

La traduzione sarà compresa, solo tredici anni più tardi e priva di corredo illustrativo, nel volume The Early Italian Poets (vedi infra), ma i soggetti avranno comunque la loro realizzazione figurativa, anche se non tutte le opere enumerate nella lettera ci sono pervenute o sono state effettivamente compiute. Riprenderemo nello specifico i singoli casi, basti per ora precisare che, dei tre disegni dichiarati "completed", è chiaramente identificabile solo il secondo, il terzo costituisce un'idea embrionale di Dantis Amor di cui resta forse traccia in un disegno preparatorio a The salutation of Beatrice (Surtees 1971: 116B, pl. 175), mentre del primo non si ha ad oggi notizia. Dei rimanenti dieci disegni progettati, sono noti soltanto: The second meeting, Dante at the Wedding-feast (dove trova forse parziale rappresentazione pure la negazione del saluto, vedi infra) e The lady of the window. Ma nel tempo altri soggetti si sono aggiunti a questi e hanno trovato celebri realizzazioni, come vedremo in dettaglio.

Segno della diffusione e della fortuna di queste opere di Dante Gabriel, e quindi in un certo senso della Vita Nuova rivisitata in chiave preraffaellita, è la raffinata edizione della Vita Nuova illustrata dai quadri di Dante Gabriel Rossetti pubblicata per i tipi Roux e Viarengo nel 1902 (e poi ristampata per tre volte, rispettivamente nel I9II, I918 e I92I, arricchita da ulteriori decorazioni grafiche e dalla prefazione di Antonio Agresti, dopo la fondazione della Società Tipografico-Editrice Nazionale, S.T.E.N., ad opera dello stesso Luigi Roux).5 Proprio da questa edizione il presente scritto intende partire, quale

3. Rinvio per questo aspetto a Hinterhäuser 1994 e Pisanti 1984.

4. Gabriele Rossetti (Vasto, I783-Londra, I854), esule a Londra per il suo appoggio ai moti degli anni '2o, poi professore d'italiano al King's College, dedicò la sua vita all'interpretazione di Dante e della figura di Beatrice. Fra i suoi studi danteschi, tutti in lingua italiana: Sullo spirito antipapale che produsse la Riforma, e sulla segreta influenza ch'esercitò nella letteratura d'Europa, e specialmente d'Italia: come risulta da molti suoi classici, massime da Dante, Petrarca, Boccaccio (Londra, Taylor, I832), Il mistero dell'amor platonico del Medio Evo, derivato da' misteri antichi (Londra, Taylor, I840), La Beatrice di Dante (Londra, Privitera, I842). Lavorò pure a un Comento analitico alla Divina Commedia, previsto in sei volumi, di cui saranno pubblicati vivente l'autore solo i primi due dedicati all'Inferno, postumo quello dedicato al Purgatorio e mai realizzato quello dedicato al Paradiso. La sua lettura di Dante è generalmente ritenuta allegorica se non addirittura esoterica, sempre in stretta connessione con gli avvenimenti storico-politici del periodo (Miralles 1989: 47). Altri interpreti la accostano alla trattatistica cinquecentesca, dal Fiorenzuola al Gelli, soprattutto per gli elementi strutturali e tematici, l'amor platonico su tutti (cfr. Circeo I984: I9I-I92).

5. Ricorderemo l'apparizione per i tipi Roux e poi Sten nello stesso torno d'anni di un'edizione delle rime di Dante, avente il titolo di Canzoniere e comprendente le poesie della Vita Nuova e altri testi poetici danteschi. Le tavole di questo Canzoniere spesso ripetono, mostrandone particolari diversi e studi preparatori, illustrazioni già presenti nella Vita Nuova, con due notevoli eccezioni: Giotto dipinge il ritratto di Dante e La barca dell'Amore. Segnaliamo inoltre l'esistenza di un'altra edizione analoga in lingua inglese che propone alcune identiche 
prima tappa di un lavoro più ampio e già avviato. L'edizione Roux non ricostruisce il progetto illustrativo (ideato già nel I848) del pittore preraffaellita, ma è allestita postuma sulla base della produzione pittorica rossettiana legata alla Vita Nuova e non senza notevoli arbitrarietà, di cui discuteremo. L'esame di questo testo e soprattutto del suo corredo illustrativo potrà tuttavia consentire di ripercorrere alcuni momenti fondamentali del libello dantesco attraverso l'opera di Dante Gabriel. Peraltro che le opere del pittore legate al libello non siano nate da una commissione editoriale né con l'esplicito obiettivo di illustrare interamente il prosimetro, se non nell'idea iniziale poi non realizzata o almeno non realizzata nei termini indicati, accresce forse i già molteplici motivi di interesse, dimostrando la pervasività dell'opera di Dante Alighieri, e della Vita Nuova in modo particolare, ${ }^{6}$ nella produzione di Dante Gabriel.

$\grave{E}$ intorno ai vent'anni che l'autore vive un momento di vera e propria infatuazione per la poesia italiana, lo stilnovo specialmente. La lettura e le prime traduzioni da Dante e dai "poeti primitivi"7 va datata alla seconda metà degli anni ' 40 , anche se la pubblicazione in volume vedrà la luce solo più tardi: nel 186I con The Early Italian Poets from Ciullo d'Alcamo to Dante Alighieri e poi nel I87I con Dante and his circle: with the Italian poets preceding him, entrambi comprendenti la traduzione della Vita Nuova (The New Life). Alla fine degli anni ' 40 , proprio come precisato nella lettera, datano anche le prime realizzazioni grafiche a penna e inchiostro (si dovrà attendere ancora qualche anno

illustrazioni: Dante Alighieri, La Vita Nuova (The New Life), translated and illustrated by photogravures after paintings by Dante Gabriel Rossetti, London, George Routledge \& Sons, s.d. Le illustrazioni qui comprese sono: The Boat of Love, From Dante's Dream, Salutatio Beatricis, Dante's Dream, Beata Beatrix, From Dante's Dream. A queste si aggiungono due illustrazioni per il quinto canto dell'Inferno incluso nel volume: Paolo and Francesca e Francesca da Rimini. Una nota nell'indice precisa che "These photogravures are produced by the special permission of Mr. Frederick Hollyer, from the photographs made by him from the original paintings of the artist". Una più recente edizione italiana (Gizzi 1985) affianca la traduzione di Rossetti ad un più ampio corredo illustrativo che riproduce (quasi) tutte le opere figurative dedicate dal poeta-pittore alla Vita Nuova.

6. Non mancano opere dedicate alla Commedia, quali Pia de' Tolomei, Beatrice nell'Eden, Lia e Rachele, o le già ricordate illustrazioni ispirate alle figure di Paolo e Francesca (per la fortuna, anche figurativa, del quinto canto dell'Inferno si veda Renzi 2007), ma i soggetti tratti dalla Vita Nuova sono, pure quantitativamente, molto più significativi, pur tenendo conto che l'intera opera dantesca (Vita Nuova, Rime e Commedia) trova spesso sovrapposizioni e intersezioni. Segnaliamo che questo lavoro si è valso del preziosissimo archivio multimediale liberamente fruibile in rete http://www.rossettiarchive.org/ (data d'accesso: novembre 20I5) che mette a disposizione testi, critica, opere figurative, cataloghi e molto altro materiale relativo a Dante Gabriel Rossetti.

7. Si conserva ancora nella sua biblioteca con le sue annotazioni il volume Poeti del primo secolo della lingua italiana, a cura di Lodovico Valeriani e Urbano Lampredi, Firenze, i8I6. Si tratta senza dubbio della fonte principale per le sue traduzioni poi incluse in The Early Italian Poets. Per la Vita Nuova l'edizione cui Dante Gabriel fa riferimento è La Vita Nuova di Dante Alighieri, a cura di Pietro Jacopo Fraticelli [III vol. delle Opere minori di Dante Alighieri], Firenze, I839, anch'essa presente nella sua biblioteca. Per le traduzioni di Dante Gabriel si vedano la sua stessa prefazione a The Early Italian Poets e Ceccarelli 2011. 
per i primi acquerelli) su temi e soggetti del libello. Dante Gabriel non manca di dedicarsi nei medesimi anni all'attività poetica in proprio, indubbiamente condizionato dalla poesia "primitiva" che legge, traduce e di cui ripropone con "apparente fedeltà" alcuni particolari, ma che, di fatto, nel complesso reinterpreta profondamente (Benedetti 1998: 7). Tale rielaborazione prenderà pure la forma di un racconto una decina d'anni più tardi, con Hand and Soul, pubblicato nel I850 sulla rivista della confraternita "The Germ" ${ }^{8}$ Vi si narra di un immaginario pittore aretino vissuto nel '200, Chiaro dell'Erma, il quale fa un percorso assai simile a quello dantesco nella Vita Nuova. Fra le molte analogie (vedi infra) è profonda ed evidente la rivisitazione: "the blessed lady" pare identificarsi con l'arte stessa e Chiaro ad un tratto capisce che "he had mistaken for faith [...] no more than the worship of beauty" (pp. 8-9). Infine ha una vera e propria visione ad occhi aperti, in cui scorge una donna "clad to the hands and feet with a green and grey raiment". La donna gli si rivolge così: "I am an image, Chiaro, of thine own soul within thee" (p. 17):

See me, and know me as I am. [...] Chiaro, servant of God, take now thine Art unto thee, and paint me thus, as I am, to know me: weak, as I am, and in the weeds of this time; only with eyes which seek out labour, and with a faith, not learned, yet jealous of prayer. Do this; so shall thy soul stand before thee always, and perplex thee no more.

Nell'estate del 1847 il protagonista dello scritto (colui che dapprima ci ha narrato la vicenda di Chiaro dell'Erma, presentandoci il tutto come veridico, citando niente meno che famosi quadri conservati a Dresda e a Monaco e immaginari studi critici) trovandosi nelle sale di Palazzo Pitti a Firenze riferisce di aver scorto un quadro: "a small one, and represents merely the figure of a woman, clad to the hands and feet with a green and grey raiment, chaste and early in its fashion, but exceedingly simple. She is standing: her hands are held together lightly, and her eyes set earnestly open. [...] On examining it closely, I perceived in one corner of the canvass the words Manus Animam pinxit, and the date 1239."

Anche senza rimarcare tutte le puntuali tangenze (dall'incontro con una donna mistica a nove anni, alla visione di Chiaro, alla febbre, dal senso di vergogna dinnanzi alla donna, alla svolta morale ecc.), la Vita Nuova è evidentemente il sottotesto ${ }^{9}$ di questo scritto, quasi manifesto di poetica tanto più

8. È la rivista letteraria dei Preraffaelliti, che ebbe breve vita (da gennaio ad aprile dello stesso I850, e mutò nome negli ultimi numeri: Art and Poetry). Cfr. la ristampa anastatica della rivista: The Germ. The Literary Magazine of the Pre-Raphaelites, Preface by Andrea Rose, Oxford, Ashmolean Museum, I992.

9. Sono state suggerite anche altre influenze: dal Sordello di Browning all' Ulalume di Poe, da Keats a Blake. Cfr. Camilletti 2003. Non andrà dimenticata neppure "l'idea di una visione di Beatrice come anima" già sostenuta da Gabriele Rossetti padre: "l'anima di Dante salita al cielo, e perciò fatta esterna sostanza separata, è quella che si chiama Beatrice”. Gabriele 
nell'intreccio fra scrittura e arte figurativa. Certo, mutatis mutandis: la donna (quasi mistica) non sarà accolta da Dio nel regno dei cieli, non sarà più simbolo della Teologia, ma piuttosto dell'Arte o direttamente espressione dell'Anima dell'artista, al contempo fonte, mezzo e fine dell'arte medesima, concezione evidentemente romantica forse già volta all'Art for art's sake. Dante quindi è radicalmente reinterpretato: ${ }^{\text {IO }}$ "Per Rossetti Amore è fascinosità intrinseca, non via. [...] è esso stesso la persona amata, che è essa stessa, poi, in qualche modo il Dio, non soltanto via a Dio. Certo Amore alligna in cuore gentile, e s'accende alla vista dell'amata; è l'Universale passare dalla potenza all'atto [...]. Ma per Rossetti l'atto ingloba immediatamente e alquanto nebulosamente tutti i sensi, la corporeità non è il 'velo' dell'anima, è parte integrante della soul, dell'anima; per cui v'è estasi dei sensi che è un'estasi dell'anima e viceversa" (Pisanti 1984: 243). Ecco che l'esperienza dantesca narrata nella Vita Nuova o da altro poeta delle origini viene riletta e attualizzata in "esperienza interiore"; "II "l'arte - la vera arte - è nella rappresentazione di sé, nell'esperienza interiore che si fa simulacro - e il simulacro ha forma di donna" (Camilletti 2005: 80).

Misureremo nel prosieguo dell'analisi, accanto ad una certa (almeno apparente) fedeltà alla lettera del testo una profonda rivisitazione in chiave neoplatonica, se non addirittura esoterica, che arriva appunto ad identificare l'immagine femminile con la sua anima. Non si dimenticherà che Dante Gabriel fu personalità fondamentale nello sviluppo del simbolismo, sia letterario sia artistico, pienamente poeta e pittore, quasi diviso fra le due pulsioni creatrici: l'inestricabile intreccio di arte e letteratura è certamente sua precipua caratteristica e come tale ineludibile nel corso della nostra analisi, tanto più che la stessa Vita Nuova è fin da principio "a double project, involving both a verbal form (the traslation) and a visual one (the series of pictures of Vita Nuova subjects)" (Becker 2004: 87). L'edizione Roux raccoglie molti dei sog-

Rossetti, La Beatrice di Dante, citato in Camilletti 2003: 86.

Io. Ricorderemo i versi (che Dante Gabriel attribuiva a Bonagiunta) che si leggono in exergo a Hand and Soul: "Rivolsimi in quel lato / Là 'nde venia la voce / E parvemi una luce / Che lucea quanto stella; / La mente mia era quella". Sono versi tratti dalla canzone adespota $\mathrm{La}$ mia amorosa mente, che tuttavia Dante Gabriel legge nel già ricordato volume del Valeriani, dove è attribuita a Bonagiunta Orbiccianti.

II. Cfr. Camilletti 2003: 80-8I. L'autore ben dimostra la sostanziale interscambiabilità fra i concetti di "mente" e "anima", correlati a livello epigrafico fra il titolo e il frammento poetico e pienamente sovrapposti nella traduzione poi offerta de La mia amorosa mente in The Early Italian Poets, dove "la mente mia" diventerà "mine own soul". Camilletti riconosce in Bonaventura da Bagnoregio l'origine di questa interscambiabilità fra mente e anima, sulla cui base Singleton ha sostenuto "I'ispirazione bonaventuriana della Vita Nuova di Dante". Si ricorderà inoltre che già Gabriele Rossetti padre, nella sua lettura in chiave politica (ghibellina e antipapale), "rilevava che Beatrice è ora la Potestà Imperiale, ora l'anima di Dante avente l'impressione di quella potestà" (Miralles 1989: 53). Cfr. inoltre Caputo 1993 e Carù 2013. 
getti (non tutti) dedicati alla Vita Nuova. Potrà forse stupire la capacità di cogliere sia i momenti più noti ed emblematici (il primo saluto di Beatrice o la negazione dello stesso, i sogni/visioni di Dante, l'apparizione della Donna gentile) sia momenti più marginali (Monna Vanna) sempre (o quasi) con costante attenzione alla lettera del testo, sia pure inevitabilmente trasfigurato in tutt'altro contesto ideologico e storico-artistico.

\section{II}

Apre il volume, precedendo il testo vero e proprio, una tavola priva di didascalia, ${ }^{12}$ raffigurante un mezzo busto di Dante (Fig. I), di profilo e a capo chino, riproduzione di uno Studio per "Il sogno di Dante", su cui torneremo, datato I87i. Il volto di Dante, come sempre nella produzione artistica di Rossetti, è derivato dal ritratto di scuola giottesca scoperto da poco tempo al Bargello. ${ }^{13}$ Entra nel vivo del libello la seconda tavola raffigurante $I l$ saluto di Beatrice (Fig. 2), ${ }^{14}$ collocata a seguire il capitolo I[III] dell'opera. ${ }^{15}$ I particolari, pure cromatici, e la scena tutta trovano fedele trascrizione nel disegno che è di fatto qui riprodotto solo per metà; la versione intera consta infatti di due parti (l'una illustra il saluto di Beatrice nella Vita Nuova, l'altra l'arrivo di Beatrice nel paradiso terrestre narrato nella Commedia, quindi il Saluto di Beatrice in cielo e in terra, come appunto recita il titolo del dipinto, cfr. Surtees I97I: II6, pl. 172). ${ }^{16} \mathrm{Si}$ trattava di pannelli decorativi - oggi alla National Gallery of Canada di Ottawa (I859, Fig. 2bis) - di un mobile della Red House di William Morris a Berkley Heath: ${ }^{17}$ "Dopo l'abbandono della casa da parte dei Morris

I2. Con poche eccezioni, ogni tavola ha un titolo e una breve didascalia, di cui daremo di volta in volta conto con l'avvertenza che sia la collocazione delle illustrazioni, sia le relative didascalie, sono frutto di scelte arbitrarie non sempre adeguate.

13. Lo stesso Agresti nella prefazione all'edizione Sten narra l'aneddoto: nel I840 il Barone Seymour Kirkup scoprì sotto l'intonaco della Cappella del Podestà del palazzo del Bargello a Firenze un frammento di affresco, che allora si attribuiva a Giotto, con il ritratto di Dante; ne fece copia e la mandò all'amico Gabriele Rossetti, esule a Londra. Non solo il ritratto rimase alla base dell'iconografia dantesca di Dante Gabriel, della Preraphaelite Brotherhood e del mondo anglosassone del tempo, ma fornì al pittore l'eccezionale soggetto dantesco già ricordato: Giotto dipinge il ritratto di Dante.

I4. La didascalia recita: "E passando per via... per la sua ineffabile cortesia... mi salutò virtuosamente tanto". Nell'edizione Sten (I92I) mutano sia il titolo (Salutatio Beatricis in terra) sia la didascalia: "Questa mirabile donna apparve a me vestita di colore bianchissimo, in mezzo di due gentil donne... e per la sua ineffabile cortesia... mi salutò virtuosamente tanto" (in entrambe le edizioni le tavole non hanno numerazione).

15. Daremo d'ora in avanti la doppia numerazione (secondo l'edizione di Gorni e di Barbi, rispettivamente) per ogni citazione della Vita Nuova. Per il testo ci atterremo invece alla lezione di Guglielmo Gorni (Gorni 1996). Segnaliamo infine che l'edizione Roux qui esaminata segue il testo di Alessandro D’Ancona (Pisa, Nistri, I872).

I6. L'edizione Roux del Canzoniere riproduce il dipinto nella sua totalità: Salutatio Beatricis in terra et in Eden.

17. Per la verità i pannelli dovevano essere tre (incompiuto quello centrale, Dantis Amor, oggi 
nel 1865 , l'opera venne rimossa e i pannelli saldati da uno spazio verticale con dipinto Amore nella mandorla, ${ }^{\mathrm{I} 8}$ la meridiana che segna l'ora della morte di Beatrice, decorata con i simboli del sole e della luna e la fiaccola abbassata in segno di lutto" (Benedetti 1998: 220). Nella cornice che inquadra e divide fra loro i due pannelli appaiono infatti diverse iscrizioni, fra cui le già ricordate parole della Vita Nuova ("Questa mirabile donna apparve a me vestita di colore bianchissimo, in mezzo di due gentil donne di più lunga etade"). Si legge inoltre sotto il pannello sinistro: "Negli occhi porta la mia donna Amore" e "Sovra candido vel cinta d'uliva, / Donna m'apparve sotto verde manto, / vestita di color di fiamma viva" (Pg. XXX 3I-33), e sotto il pannello destro, ancora: "Guardami ben: ben son, ben son Beatrice" (Pg. XXX 73). Al centro appare la mandorla che racchiude la figura di Amore con in mano la meridiana ("L'ora che lo suo dolcissimo salutare mi giunse, era fermamente nona di quello giorno") e la data "9 JUN: I290", e sotto si legge: "Quomodo sedet sola civitas", citazione dalle Lamentazioni di Geremia con cui, come noto, si apre il cap. 2I [XXX] (vedi infra). La morte di Beatrice segna il momento di passaggio tra la terra e il cielo, i due momenti del dipinto, appunto. Ma l'ora nona è anche quella del saluto di Beatrice, come sarà pure l'ora del sogno premonitore della morte di lei. Se l'aspetto "numerologico" (non senza suggestive ipotesi di corrispondenze metriche, cfr. Gorni 1996: xxiv-xxv) è fra quelli più rilevanti per Rossetti padre, come per tutta la critica anglosassone, Dante Gabriel non fa eccezione, come si evince dall'iterazione quasi ossessiva della meridiana e della data nei dipinti o nella cornice. La prima scena riproduce abbastanza fedelmente la situazione delineata nella Vita Nuova: Beatrice appare "in mezzo a due gentili donne, le quali erano di più lunga etade". ${ }^{19}$ Non è casuale che Dante e Beatrice, nel primo disegno posizionati sullo stesso livello, siano poi rappresentati nel loro incontro su una scala: le tre donne e quindi Beatrice scendono, sale invece Dante. L'idea della scala introduce un certo dinamismo (si osservi tra l'altro che nessuno dei tre personaggi è perfettamente al livello di un altro) ed è una felice rappresentazione dell'ascesa del poeta, attraverso l'azione beatificante del saluto di lei. Il profilo della città che appare alle loro spalle andrà convenzionalmente identificato con Firenze (il corrimano della ringhiera su cui Dante poggia la mano sinistra mostra evidentemente

alla Tate Gallery, vedi infra). Il soggetto è molto ricorrente nella produzione di Dante Gabriel, dai primi disegni datati alla fine degli anni '40, fino ad anni più tardi ('70-'80) dove muta decisamente l'iconografia: Beatrice a figura quasi intera con la città sullo sfondo, dietro di lei in lontananza si intravedono Dante e Amore; quest'ultimo seduto su un pozzo, abbraccia Dante con le sue ali (cfr. Fig. 6bis).

I8. Amore nella mandorla che congiunge i due dipinti è probabilmente derivato dal disegno dato per finito nella lettera già ricordata (Fredeman 2002-2010: 76).

19. Sempre Maria Teresa Benedetti informa che "le tre figure femminili corrispondono a Red Lyon Mary (la padrona di casa dello studio Red Lyon Square), Jane Morris e Fanny Cornforth" (Benedetti 1998: 220). 
due gigli). ${ }^{20}$ Dante è vestito di rosso (il colore dell'amore) mentre Beatrice ha una veste non propriamente candida, si intravedono disegni con colori tenui su fondo bianco: dunque non è esattamente "vestita di colore bianchissimo" ("pure white" nella traduzione di Rossetti). ${ }^{2 \mathrm{I}}$ Il dato cromatico è invece pienamente rispettato nel secondo pannello che illustra l'incontro di Dante e Beatrice nel paradiso terreste $(P g . \mathrm{XXX})$. Non dovrà stupire l'apparente contraddizione fra minuto realismo e forte simbolismo. La componente realista, in particolare, è stata ben messa a fuoco nella recente mostra tenutasi a Torino: "il gruppo creò un linguaggio realista assolutamente originale, preciso, vivido e senza mezzi termini" che, guardando oltre l'apparente contraddizione, trae origine dalla fotografia (Barringer 20I4: 16).

Altro momento cruciale che si reitera nell'opera pittorica di Rossetti è la negazione del saluto: Beatrice nega il saluto a Dante (Fig. 3). Arbitraria la collocazione della tavola a seguire il sonetto Cavalcando l'altrier per un cammino (cap. 4[IX]), riferendosi il testo a un episodio relativo allo "schermo" che di fatto è precedente la negazione del saluto (cap. $5[\mathrm{X}]$ ), anzi ne costituisce proprio l'antefatto poiché Dante "simulò un amore così appassionato" (questa l'interpretazione di Gorni per "la feci mia difesa tanto") che "troppa gente ne ragionava oltre li termini della cortesia". Non del tutto corrispondente all'iconografia dell'opera neppure la didascalia (“...quella gentilissima... passando per alcuna parte mi negò il suo dolcissimo salutare"): l'immagine infatti non illustra il capitolo $5[\mathrm{X}]$, peraltro avaro di particolari figurativi (sappiamo solo che Beatrice "passando per alcuna parte mi negò il suo dolcissimo salutare") e comunque non restituiti da Rossetti neppure nella loro genericità, dato che la scena è ambientata in un luogo molto più caratterizzato di quanto il passo citato suggerisca. Tutto porta quindi a ritenere che l'illustrazione, nonostante il titolo, ${ }^{22}$ faccia riferimento all'episodio del gabbo, ossia al cap. 7 [XIV]. Qui Dante è condotto da una persona amica "in parte ove molte donne gentili

20. Sarà opportuno ricordare che Dante Gabriel non visitò mai l'Italia, ma è documentata la sua conoscenza di Firenze, e non solo, attraverso stampe e fotografie. Senza contare l'influsso dell'Arundel Society, che promosse la diffusione di riproduzioni (cromolitografie, a colori quindi) dell'arte italiana cosiddetta "primitiva", non vanno dimenticate altre fonti di conoscenza dei luoghi e dell'arte italiana. Prima di fondare la confraternita, già vicino a William Hunt ed Everett Millais, frequentò con loro The Cyclographic Society, un piccolo club di disegnatori, dove probabilmente conobbe le incisioni ottocentesche degli affreschi del camposanto di Pisa ad opera di Paolo Carlo Lasinio (I8I2). Cfr. gli interventi di Bonetti 20II: 176-I83 e Marini 20II: 184-196.

2I. Sul colore bianco (e sul mutamento cromatico rispetto al primo incontro) rinvio al commento di Gorni (Gorni 1996: 7 e 15) che intravede nel reiterarsi del bianco (colore sia della veste in cui appare Amore dopo la negazione del saluto da parte della gentilissima, sia del velo con cui le pie donne coprono il capo di Beatrice defunta, sia della "nebuletta" con cui l'anima di Beatrice ascende al cielo) "un'allusione angelica", da leggersi addirittura come una riscrittura della Trasfigurazione di Cristo (De Robertis 1980: 36).

22. Come già anticipato commentando la lettera a Charles Lyell, l'idea di questo soggetto nasce "autonoma" e solo in seguito si sovrappone, almeno nel titolo, alla negazione del saluto. 
erano adunate". Si spiega poi che le donne erano riunite insieme, secondo l'usanza, in compagnia di una gentil donna appena sposata "nel primo sedere alla mensa, che facea nella magione del suo novello sposo". Dante tuttavia è colto da "uno mirabile tremore" che comincia nella parte sinistra del suo petto per estendersi su tutto il suo corpo, tanto da costringerlo, proprio al fine di dissimulare il suo stato, ad appoggiarsi "ad una pintura, la quale circundava questa magione". Ė solo a questo punto che Dante riconosce tra loro "la gentilissima Beatrice". Rossetti rappresenta una scena molto ampia, quasi affollata di personaggi, restituendone efficacemente l'effetto quasi teatrale, già osservato da Gorni nel testo dantesco e forse qui acuito dall'impiego di colori particolarmente vividi, come vediamo nell'acquerello (Fig. 3bis) eseguito nel I85I da cui è tratta la riproduzione. Ne esiste una seconda versione, conservata a Oxford, che presenta alcune varianti; restano tuttavia identici i colori più intensi: il verde e il blu degli abiti femminili al centro della composizione, insieme con il rosso della veste di Dante e l'oro dell'abito della bambina che porge fiori a Dante (mentre muta la veste dell'amico, rossa e verde nella prima versione, nera e dorata nella seconda, non riprodotta nel presente articolo). Dante e l'amico stanno in piedi, di profilo, sulla destra del quadro, la parte bassa e centrale resta libera, il suolo è cosparso di immancabili fiori, mentre la figura di Beatrice (nei cui tratti è riconoscibile Elizabeth Siddal) sta esattamente al centro dell'immagine. Sulla sinistra, intorno a Beatrice si affollano molte figure, sempre e solo donne accanto a lei, mentre alcuni uomini si scorgono nella parte più alta della scena, dove appaiono pure gli sposi (invenzione di Dante Gabriel), rappresentati con fattezze diverse nelle due versioni. Un certo dinamismo è garantito dai diversi "livelli" previsti nella composizione, $\mathrm{i}$ gradini più bassi su cui sale l'amico di Dante, il piano occupato da Beatrice, da Dante stesso (i due sono ora sullo stesso livello) e da alcune donne, la scala da cui scendono donne e uomini e ancora sulla sinistra la balaustra della scala avvolta fra tralci d'uva che tre figure dal basso si accingono a tagliare. Pure il gioco fra interno ed esterno conferisce ulteriore dinamismo alla scena: le figure femminili infatti stanno entrando nella casa, dal cui ingresso si intravvede un balcone dove si sporgono altre figure di suonatori. Cambia pure fra le diverse versioni la "quinta" che chiude la scena, come rafforzata nella sua funzione di cornice con l'acquerello del I855, dove la casa appare circondata da un muro che si apre solo con un piccolo tondo verso l'esterno. Fedele qui alla lettera del testo, Rossetti rappresenta la "pintura" cui Dante si appoggia per dissimulare il tremore, letta dai commentatori ora come affresco ora come arazzo (intendendo di conseguenza "magione" come l'interno della casa). Rossetti con ogni evidenza intende affresco (nella traduzione leggiamo infatti "painting") e vi rappresenta una serie di figure angeliche che si distinguono per il bianco delle vesti, l'oro delle aureole, il biondo dei capelli, il tenue colore rosato delle ali. 
Il pensiero (o la visione) della morte di Beatrice è uno dei soggetti più praticati, non privo di affinità, come gli interpreti non mancano mai di ricordare, con il dato biografico: la morte prematura di Elisabeth Siddal, da contestualizzare nel complesso e tormentato rapporto fra i due. L'edizione Roux presenta due illustrazioni per questo momento: Gli angeli portano al cielo l'anima di Beatrice (Fig. 4) e Il sogno di Dante (Fig. 5). La prima altro non è che uno studio per la seconda: ${ }^{23}$ la riproduzione è tratta infatti da un disegno preparatorio per Il sogno di Dante, precisamente uno studio per lo spirito di Beatrice circondato dagli angeli, conservato in una collezione privata (un gesso nero con tocchi di rosso su carta grigio-verde, di piccolo formato, datato I870). Il disegno raffigura un gruppo di angeli, distinguibili per le ali, in cerchio attorno alla nuvoletta ("an exceedingly white cloud", nella traduzione di Dante Gabriel), ${ }^{24}$ così come appaiono nella parte alta del più ampio Dantés Dream. La "nebuletta bianchissima" rappresenta, secondo gli interpreti, l'anima di Beatrice. ${ }^{25}$

La monumentalità del dipinto Dantés Dream (oltre due metri per tre), un olio commissionato da W. Graham - che poi lo rifiutò per l'evidente squilibrio tra soggetto e formato -, oggi alla Walker Art Gallery di Liverpool (Fig. sbis), e la proliferazione di studi preparatori - senza contare il precedente acquerello della Tate Gallery (Il sogno di Dante al tempo della morte di Beatrice, I859, cfr. Surtees 1971: 8I, pl. 95), che presenta analogo impianto compositivo $-{ }^{26}$ rendono conto già di per sé del lungo lavoro dedicato al soggetto. Siamo sempre al capitolo I4 [XXIII] dell'infermità di Dante, seguita dal sogno/ visione della morte di Beatrice. ${ }^{27}$ I papaveri sul pavimento, i ricchi colori degli

23. Il Sogno di Dante è pervasivo pure nella già ricordata edizione del Canzoniere (ben cinque gli studi preparatori riprodotti, oltre al Dante's Dream vero e proprio, unica immagine riprodotta a colori e su due pagine). Due studi e lo stesso Dante's Dream compaiono anche nell'edizione inglese Routledge, già ricordata.

24. L'edizione Roux non ha didascalia, mentre nella successiva edizione Sten si legge: “...pareami vedere moltitudine di angeli i quali tornassero in suso ed avessero d'inanzi da loro una nuvoletta bianchissima..." La tavola segue la prima parte del capitolo i4 [XXIII] quando la "dolorosa infermitade" porta Dante a riflettere sulla caducità della vita (propria e altrui) e quindi a immaginare, con una visione dalla scoperta eco biblica, il giungere della ferale notizia.

25. La nuvoletta è interpretata come "l'anima di Beatrice (Casini) o l'Ascensione di lei (Singleton)" (cfr. Gorni 1996: 127). L'ascensione di Beatrice fra gli angeli si aggiungerebbe agli altri elementi che già rendono esplicito il parallelismo fra Beatrice e Cristo: dal terremoto agli altri prodigi fino all'Osanna in excelsis, acclamazione che accompagna l'ingresso di Gesù a Gerusalemme.

26. La nuova versione muta i colori e le vesti, con ampi e morbidi drappeggi, aggiunge elementi simbolici e cambia volto a Beatrice - Jane Morris invece di Elizabeth Siddal. Mutano inoltre lo sfondo di sinistra e la parte alta con gli angeli attorno alla nuvoletta.

27. La didascalia recita: "Mi condusse a veder mia donna morta: E quando io l'ebbi scorta, vedea che donne la covrian d'un velo". L'illustrazione è collocata fra le pagine di Donna pietosa e di novella etade, fra la seconda e la quarta stanza, ma per la verità è la quinta stanza a costituire il soggetto del dipinto: "[...] Allor diceva Amor: - Più nol ti celo; / vieni a veder nostra donna che giace. - / Lo ymaginar fallace / mi condusse a veder madonna morta; / e quand'io l'avea scorta, / vedea che donne la covrian d'un velo; / ed avea seco umiltà verace, I che parea che dicesse: - Io sono in pace -." 
abiti e della composizione tutta, la morbidezza delle forme, dei panneggi delle vesti soprattutto, finanche l'espressione quasi sospesa delle figure danno vita ad un mondo visionario e sognante. Il simbolismo cromatico si ripete, il verde delle pie donne, il bianco di Beatrice, il rosso di Amore. Il testo dantesco precisa che il velo copre solo la testa di Beatrice (cioè esclusivamente la sommità del capo, proprio perché rimanesse visibile il volto, come generalmente si è interpretato, a cominciare da Barbi che ne ha rilevato la conformità con l'uso del tempo). Nel dipinto invece il velo sta per coprire tutto il corpo di Beatrice, ma essendo colto nel momento in cui le Pie donne lo stanno apponendo, il volto di Beatrice è ben visibile.

L'illustrazione che ha meno a che vedere con il testo dantesco è paradossalmente quella riferita ai versi più noti del libello, puntualmente riportati nella didascalia: "Tanto gentile e tanto onesta pare / la donna mia, quand'ella altrui saluta, / ch'ogne lingua deven tremando muta, / e li occhi no l'ardiscon di guardare." Intitolata semplicemente Beatrice (Fig. 6) e collocata a seguire la parte in prosa del capitolo ${ }_{17}[\mathrm{XXVI}]$ riprende sostanzialmente titolo e soggetto già esaminato nella Fig. 2 (Il saluto di Beatrice). Muta però decisamente l'iconografia (siamo in anni più tardi '70-'80). Esistono diversi studi che precedono il dipinto a olio oggi conservato al Museum of Art di Toledo (Ohio). La riproduzione inserita nell'edizione Roux è derivata da uno di questi studi che rappresenta solo il volto della donna (o meglio il volto e le spalle), perdendo così alcuni riferimenti visibili nel dipinto intero che pure "tradisce" alcuni punti essenziali del testo dantesco: Beatrice (Jane Morris) occupa quasi tutto lo spazio della tela con la sua veste bianca e lievemente dorata, attorno a lei fiori nella parte bassa del quadro, sullo sfondo una città. ${ }^{28}$ Sulla destra in lontananza si intravedono Dante e Amore, in abito rispettivamente blu e rosso; Amore seduto su un pozzo, abbraccia Dante con le sue ali (esiste uno studio di queste due figure, conservato al British Museum di Londra, che consente l'identificazione). Manca l'essenziale dimensione "sociale" del saluto (o anche solo del semplice passaggio) di Beatrice che appare sola nella città, non fosse per la presenza defilata del poeta e di Amore. Naturalmente questo tratto tipicamente stilnovista è ormai lontano dal sentire preraffaellita che, come stiamo osservando, tende a caricare di significato simbolico la sola figura di Beatrice.

Ancora sui generis, ma per ragioni diverse, pure la successiva illustrazione tratta da un'opera molto nota: Beata Beatrix (Fig. 7). L'opera si distingue per

28. I particolari architettonici sono stati ricavati da fotografie fornite da Farfaix Murray e si riferiscono probabilmente a Siena (Benedetti 1998: 344). Ricordiamo che nei volti delle donne rossettiane si scorge l'ombra del grande ritratto rinascimentale italiano (da Leonardo a Raffaello) giunto a lui attraverso The italian school of design di William Joung Ottley (1823). 
un'atmosfera decisamente onirica, quasi un unicum nel corpus rossettiano (Barringer 20I4: 198), dove i colori non risultano freddi e brillanti come di consueto, bensì attenuati e sfumati come il trattamento complessivo della tela, che "seems to trace an evenescent film over the canvas" (Becker 2004: 80). Queste caratteristiche trovano ragione nella datazione: il quadro fu inziato prima del I862 - anno della morte di Elisabeth Siddal, qui ritratta quale Beatrice -, poi ripreso nel 1864 e infine terminato solo nel 1870 . Non è la prima volta che le due figure, Beatrice e Lizzie, oscillando "tra suggestioni mistiche e abbandoni sensuali" (Gizzi 1999: 53), si sovrappongono nella pittura rossettiana, tanto più dopo la morte - probabilmente, come noto, un suicidio - della donna.

Il titolo è una citazione esplicita dalla Vita Nuova. Se il nome di Beatrice si rivela cruciale fin dal principio dell'opera dantesca ("la quale fu chiamata da molti Beatrice li quali non sapeano che si chiamare"; cap. I[II]) il sintagma "Beatrice beata" fa la sua comparsa solo al capitolo I9 [XXVIII] cioè esattamente nel momento in cui "lo segnore della giustizia chiamoe questa gentilissima [...] Beatrice beata". Ed è proprio tutta questa parte della Vita Nuova (con particolare attenzione al capitolo $\left.{ }_{19}[X X V I I I-X X X]\right)^{29}$ che a nostro avviso la presente opera di Rossetti intende rappresentare, sebbene dicano concordemente i commentatori, anche sulla scorta delle parole dello stesso autore: "The picture must of course be viewed not as a representation of the incident of the death of Beatrice, but as an ideal of the subject, symbolized by a trance or sudden spiritual trasfiguration. Beatrice is rapt visibly into Heaven, seeing as it were through her shut lids (as Dante says at the close of the Vita Nuova) "Him who is blessed throughout all ages'" (Fredeman 2002-20IO: VI, 88-89). Nella stessa lettera a William Graham (datata II marzo I873) Dante Gabriel spiega alcuni simboli: "the radiant bird, a messenger of death, drops the white poppy between her open hands" mentre sullo sfondo (quasi risucchiato dall'ombra, figura spettrale, come la stessa Beatrice) "Dante himself is seen to pass gazing towards the figure of Love opposite, in whose hand the waning life of his lady flickers as a flame" (Ibidem). Possiamo individuare altri elementi, il pozzo e l'albero (questo effettivamente non distinguibile nella riproduzione bianco e nero dell'edizione Roux), entrambi simboli di rinascita. Certamente non si intende rappresentare la morte di Beatrice - peraltro non narrata, se non in forma di visione (capitolo I4[XXIII]), neppure nel libello dantesco, anzi Dante insiste sulla scelta: "non è lo mio intendimento di trattarne qui" -, ma il dipinto illustra quasi letteralmente i capitoli legati alla morte di Beatrice e non tanto il successivo cap. 20 [XXXI] come parrebbe indicare la didascalia.

29. La tavola in effetti segue proprio il cap. 19[XXVIII-XXIX] nell'edizione Roux. La didascalia recita: "Si che dolze disire / Lo giunse di chiamar tanta salute / E fe' la di qua giuso a sé venire: / Perché vedea ch'esta vita noiosa / Non era degna di sì gentil cosa" (Li occhi dolenti per pietà del core, vv. 24-28). 
La meridiana ferma sulle nove ("ne la sua partita cotale numero pare che avesse molto luogo") illustra alla lettera il capitolo ${ }_{19}$ [XXIX], dove non solo si osserva che proprio nel momento della morte della gentilissima il nove ricorreva per diversi motivi nel calendario arabo, siriaco e cristiano, ma si dichiara che il nove "fu tanto amico" di Beatrice perché "nella sua generazione tutti e nove li nobili cieli perfettissimamente s'aveano insieme" e soprattutto "questo numero fue ella medesima”. Alle spalle di Beatrice (e della meridiana) appoggiata sulla balaustra del balcone che divide in due lo spazio del quadro, si distinguono elementi propri della città (case, tetti, un pozzo) che è forse identificabile in Firenze visto il ponte che si intravede sullo sfondo: naturalmente potrebbe essere Ponte Vecchio, ma si è pure evocato il Tamigi e il Battersea Bridge, facendo prevalere l'aspetto autobiografico. Eppure la città di Firenze risulta chiamata in causa in questi stessi capitoli, dapprima introducendo la morte di Beatrice con la nota citazione dalle Lamentazioni di Geremia: "Quomodo sedet sola civitas plena populo! facta est quasi vidua domina gentium", ripresa poi nel capitolo $20[\mathrm{XXX}]$. Queste stesse parole sono peraltro riprese nella parte bassa della cornice del dipinto oggi conservato alla Tate Gallery di Londra (ma ne esistono numerose repliche). Come la Lamentazione conferisce un tono liturgico a questa parte del racconto di Beatrice, così il dipinto annuncia nella cornice il suo aspetto per così dire sacrale. Benedetti legge il quadro come "compiuta rappresentazione" del rapporto fra Amore e Morte. Beatrice-Lizzie diventerebbe "l'oggetto del desiderio, trasfigurato" incarnando "un'aspirazione all'infinito". Sulla scorta del filosofo svedese Emmanuel Swedenborg che sosteneva "come l'unione dei due sessi fosse esperienza religiosa, in grado di avvicinare all'amore di Dio", per Benedetti "il volto di Beatrice istituisce un legame fra eros e trascendenza, orgasmo e rivelazione" (Benedetti I998: I0).$^{30}$ I colori sono simbolici: sempre rosso e verde per Dante e Beatrice, rispettivamente il rosso colore della morte, il verde colore della vita. La luce è diffusa, ma più intensa sullo sfondo e sulla meridiana. Attorno al capo di Beatrice pare formarsi un'aureola di luce.

Dante che disegna un angelo in memoria di Beatrice (Fig. 8) ${ }^{31}$ è uno dei soggetti più ricchi ispirati alla Vita Nuova. La tavola segue il cap. 23[XXXIV] come esattamente riportato nella didascalia (“...io mi sedea in parte, ne la quale, ricordandomi di lei, disegnava uno angelo sovra certe tavolette...”). La riproduzione non rende neppure lontanamente la forza visiva (tutta data dal forte cromatismo) che si percepisce davanti all'acquerello (Fig. 8bis), di di-

30. "Le idee di Swedenborg, a lui pervenute attraverso Blake e Browning, sono familiari anche a Baudelaire e a Mallarmé: il concetto simbolista delle corrispondenze fra arte, musica e letteratura trova un parallelo ideale nell'analisi swedenborghiana delle corrispondenze fra elementi terreni e celesti, fra spirito e materia" (Benedetti I998: I2).

31. Sul valore iconico e l'idea di visione in questo episodio della Vita Nuova, si rinvia a Ciccuto I994. 
mensione insolitamente notevole $(\mathrm{mm} .420 \times 6 \mathrm{IO})$, conservato all'Ashmolean Museum di Oxford. L'acquerello, datato I853, valse al suo autore il primo forte apprezzamento di John Ruskin.

La scena è arbitrariamente collocata in un interno ${ }^{32}$ dove il poeta è sorpreso da alcune persone intento a disegnare. Dante, vestito di nero, con le sembianze del fratello del pittore, William, è inginocchiato davanti alla finestra che si apre sull'Arno e Firenze. Sopra il davanzale tre bottiglini di colore (giallo, rosso e blu) insieme con un melograno, sotto vari oggetti fra cui un libro e un liuto. Sopra la finestra, appena scostata dalla tenda, una clessidra, e seguendo il perimetro della stanza nella parte più alta si incontrano nell'ordine, un giglio bianco, uno specchio, un'immagine della Madonna col Bambino e una serie di teste angeliche nei peducci degli archi. La porta della stanza apre su un prato e un bosco, da una parte la città dunque e dell'altra uno scenario naturale, edenico. Il disegno di Dante è ben visibile e rappresenta una testa di donna aureolata nella quale ancora una volta si riconoscono le sembianze di Elisabeth Siddal. Le tre figure astanti occupano la parte centrale del quadro: un giovane uomo che appoggia la sua mano sulla spalla di Dante e un poco discosti un altro uomo più anziano che cerca di trattenere il giovane e la donna che si appoggia alla spalla del vecchio in un gesto di partecipazione emotiva alla scena che sta osservando. I colori vividi, che ricordano gli smalti medievali e i codici miniati, creano un'atmosfera intensamente poetica. Non manca l'ispirazione a modelli fiamminghi e nord europei, con prelievi da Dürer (l'acquamanile), da Memling (lo specchio ovale), da Van Eyck (non andranno dimenticati i viaggi nei Paesi Bassi, dove Dante Gabriel restò particolarmente impressionato dalla pittura fiamminga), ma vi circola anche un clima che ricorda il Quattrocento italiano da Ghirlandaio a Lippi a Botticelli (Benedetti 1998: 178).

Penultima riproduzione inclusa nell'edizione Roux è La donna della finestra (Fig. 9). Nella didascalia leggiamo: "Color d'amore e di pietà sembianti. Non preser mai così mirabilmente viso di donna..." Il sonetto è peraltro leggibile, nella nostra edizione, nella pagina sinistra accanto all'illustrazione e rappresenta l'intero episodio della donna gentile o donna pietosa, reinterpretata nel Convivio come allegoria della filosofia (capp. 24-25[XXXV-XXXVI]). Sulla cornice dorata del quadro conservato al Fogg Art Museum di Cambridge (Massachussets), qui riprodotto, si legge la trascrizione (e relativa traduzione) del sonetto Videro gli occhi miei quanta pietate con l'indicazione "Vita Nuova di Dante". L'opera fu eseguita nel I879, ma diversi studi preparatori datano all'inizio degli anni 70. Il "colore palido" della donna è uno dei tratti salienti

32. Nel testo dantesco l'ambientazione non è precisata ("io mi sedea in parte") e pure la traduzione di Rossetti non aggiunge nulla ("I sat alone"), ma la circostanza lascia intendere che non si tratta di un interno visto che Dante non si accorge subito della presenza di alcuni uomini (non donne e uomini come nell'acquerello) che "riguardavano quello che io facea". 
e del testo dantesco ${ }^{33}$ e della tela di Rossetti che rappresenta un'insolita Jane Morris con i capelli rossi. L'immagine illustra infatti il momento cruciale del cap. $24[\mathrm{XXXV}]$, quando "una gentile donna giovane e bella molto" guarda Dante "sì pietosamente, quanto a la vista, che tutta la pietà parea in lei accolta”. Pure il cap. $25[\mathrm{XXXVI}]$, già richiamato dalla didascalia, gioca una parte importante con il suo forte valore visivo che sembrerebbe quasi preludere all'ecfrasi se l'aspetto descrittivo non finisse sempre per restare inappagato nel libello dove paradossalmente si esalta il senso della vista senza descrivere, in particolare proprio nei già citati versi "Color d'amore".

Chiude il volume Dantis Amor (Fig. Io), la più astratta sintesi dell'opera dantesca che Dante Gabriel abbia concepito, oltre che estrema elaborazione dell'idea di amore alla base della sua arte, vera e propria celebrazione della potenza dell'amore. Ovviamente l'opera si colloca su una "linea fantastico-visionaria" rispetto alla linea più realista che costituisce pure un tratto distintivo del preraffaellismo e dello stesso Rossetti. La didascalia recita: "Quand'egli è giunto là, dove 'l desira, vede una Donna, che riceve onore e luce sì, che par lo suo splendore lo peregrino spirito la mira". Si tratta dei versi 5-8 dell'ultimo sonetto della Vita Nuova, appena prima della "mirabile visione" conclusiva. L'illustrazione è collocata dopo la spiegazione del sonetto "Oltre la spera". La migliore didascalia dell'opera viene tuttavia dalle iscrizioni che vi si leggono all'interno, tratte proprio dalle ultime righe del libello, dopo aver espresso il noto proposito di "dicer di lei quello che mai non fue detto d'alcuna":

E poi piaccia a colui che è sire de la cortesia, che la mia anima se ne possa gire a vedere la gloria della sua donna, cioè di quella benedetta Beatrice, la quale gloriosamente mira ne la faccia di colui qui est per omnia saecula benedictus.

La riproduzione deriva da uno studio a penna e inchiostro per il più noto Dantis Amor della Tate Gallery, olio su pannello (non finito) originariamente parte del mobile della Red House di William Morris (lo stesso del Saluto di Beatrice, già esaminato). Al centro Amore, alato, in abiti da pellegrino, con in mano arco e frecce e una meridiana con impressa la solita data del 9 giugno I290, si staglia su uno sfondo diviso diagonalmente: a sinistra in alto il volto di Cristo in un tondo (un sole) da cui si irradiano raggi di fuoco, a destra in basso il volto di Beatrice incorniciato da una mezza luna su uno sfondo di stelle. Questo disegno ha alcune differenze rispetto all'olio più noto (Surtees 197I: II7, pl. I79) ed è unanimemente definito di più alta qualità, tanto che si è dubitato che il pannello del mobile sia stato effettivamente dipinto da Rossetti.

33. Color d'amore rinnova la tradizione topica del pallor amantium (che ha come noto origine dall'ovidiano Palleat omnis amans). Per tutte le dovute distinzioni, anche cromatiche, tra Beatrice e la Gentile si veda Bertolucci 1989: 192-193. Per il corretto significato di "pallido" si rinvia a Feo 1975: 322-324. 
Nella diagonale che separa lo sfondo leggiamo: "Lamor che muove il sole e l'altre stelle", mentre nella mezza luna che incornicia Beatrice si legge "Quella beata Beatrice che mira continuamente nella faccia di colui". Nel sole in cui appare il volto di Cristo è posta l'iscrizione: "Qui est per omnia saecula benedictus". Nell'olio mutano le vesti di Amore e lo sfondo, i colori sono particolarmente vividi, domina il giallo oro. Scompaiono le iscrizioni con l'eccezione di quella attorno al volto di Cristo. Resta identica la simbologia riconoscibile nella posizione quasi speculare di Cristo e Beatrice, rispettivamente sole e luna, cioè "luce e oscurità, attivo e passivo, cielo e terra, maschile e femminile [...]. Sono immagini frequenti nelle incisioni per le edizioni tardo cinquecentesche della Divina Commedia, certamente note a Rossetti. [...] L'artista rappresenta inoltre il contrasto degli opposti: la luna come riflesso ideale della luce del sole: Beatrice come riflesso di Cristo" (Benedetti 1998: 222). Benedetti considera inoltre Dantis Amor come una prefigurazione di Beata Beatrix: "Rossetti ha creato un diagramma araldico della scomparsa della donna e della sua unione con Cristo. ${ }^{34}$ Eliminato ogni aspetto narrativo, l'immagine è ridotta a rappresentazione schematica di rapporti essenziali, per lui riconducibili al significato centrale degli scritti danteschi: il potere dell'amore come manifestazione del Creatore, espresso da Dante nel verso finale della Commedia 'l'amor che move il sole e l'altre stelle"' (Benedetti I998: II).

La Vita Nuova, quindi, è per Dante Gabriel un testo cruciale. Attentamente meditato attraverso la traduzione e quasi onnipresente nella sua produzione artistica, è un'inesauribile fonte di soggetti per i suoi quadri, senza contare che il libello dantesco è senz'altro fonte per Hand and Soul, scritto che molto rivela della sua rivisitazione dell'opera. Rossetti rispetta apparentemente particolari, situazioni, momenti (non mancano tuttavia le differenze, come si è visto); insiste su alcuni punti fondamentali, il concetto di visione su tutti. Eppure, senza soluzione di continuità e senza contraddizione, Dante è profondamente rivisitato, arricchito di esoterismo, in chiave neoplatonica, e di simbolismo, segno di quanto e di come il libello sia stato vitale in questo contesto culturale.

34. Le analogie e i parallelismi fra Beatrice e la figura di Cristo sono stati via via rimarcati e continuano ad essere ribaditi pure in sede critica, non ultimo andrà ricordata pure l'analisi dell' "analogia cristologica" recentemente offerta da Marco Santagata (Santagata 20II: 2II2I2). 


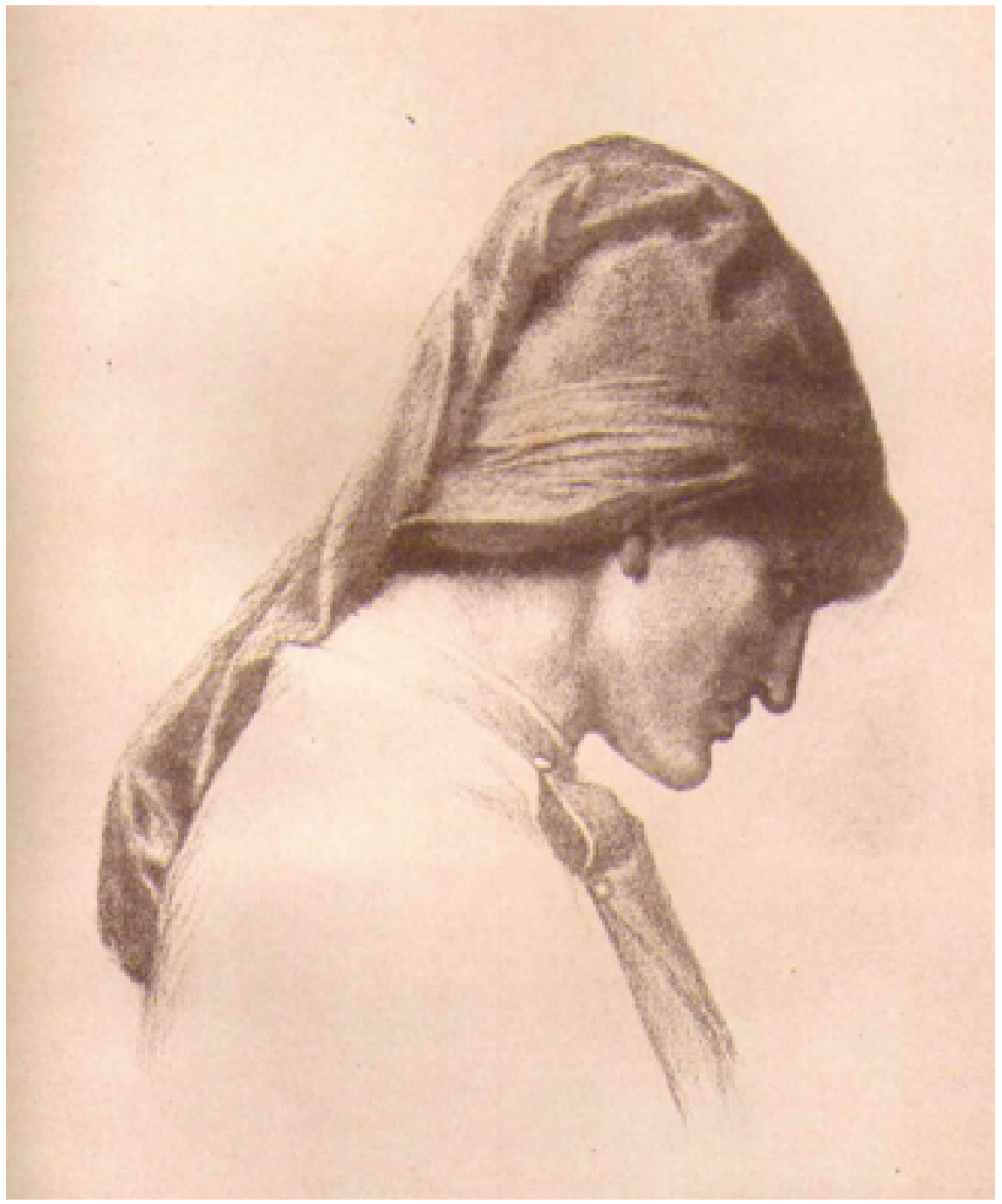

Fig. I: D. Alighieri, Vita Nuova illustrata dai quadri di Dante Gabriel Rossetti, Roux e Viarengo, Torino, 1902 


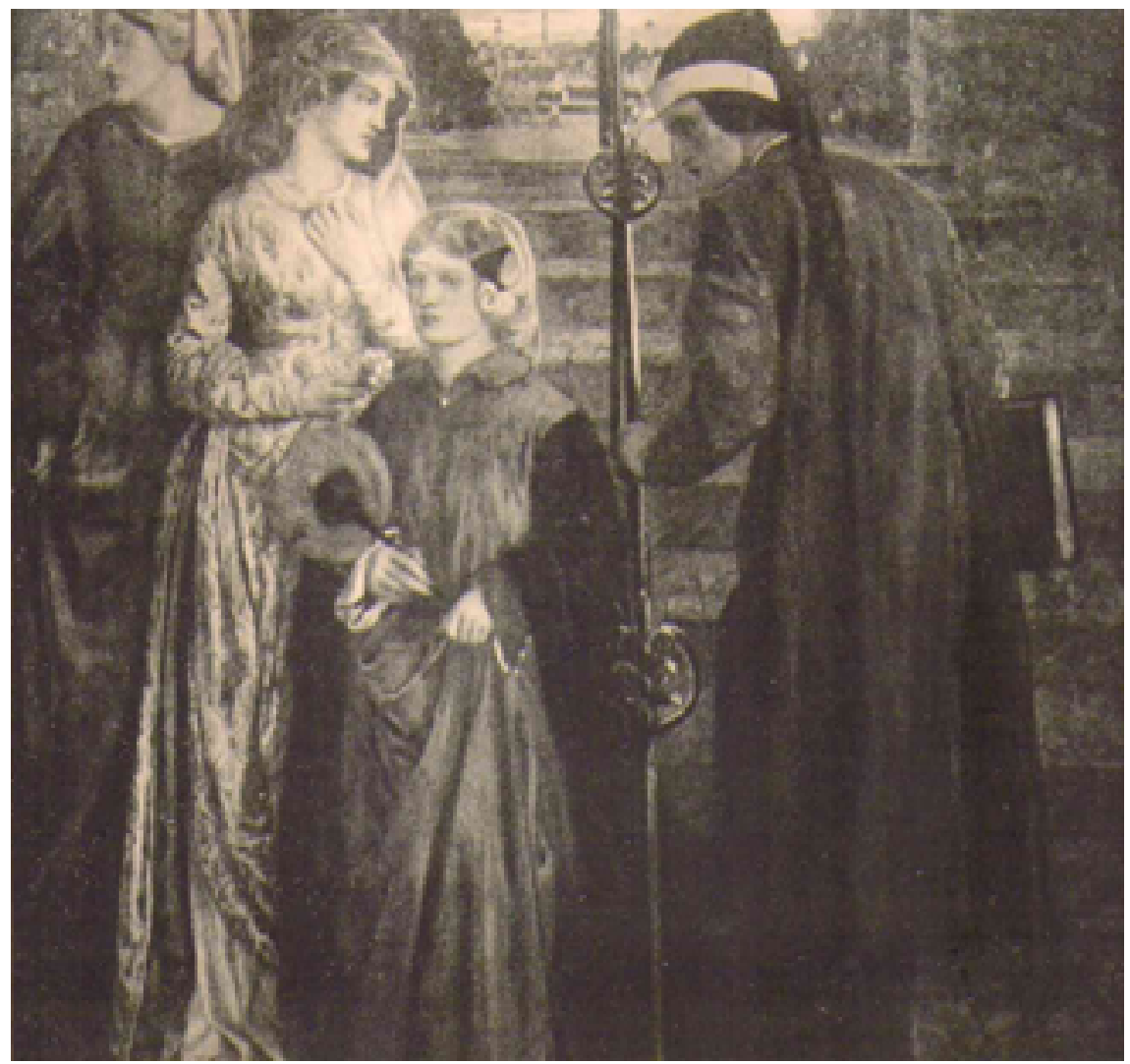

Fig. 2: D. Alighieri, Vita Nuova illustrata dai quadri di Dante Gabriel Rossetti, Roux e Viarengo, Torino, 1902.

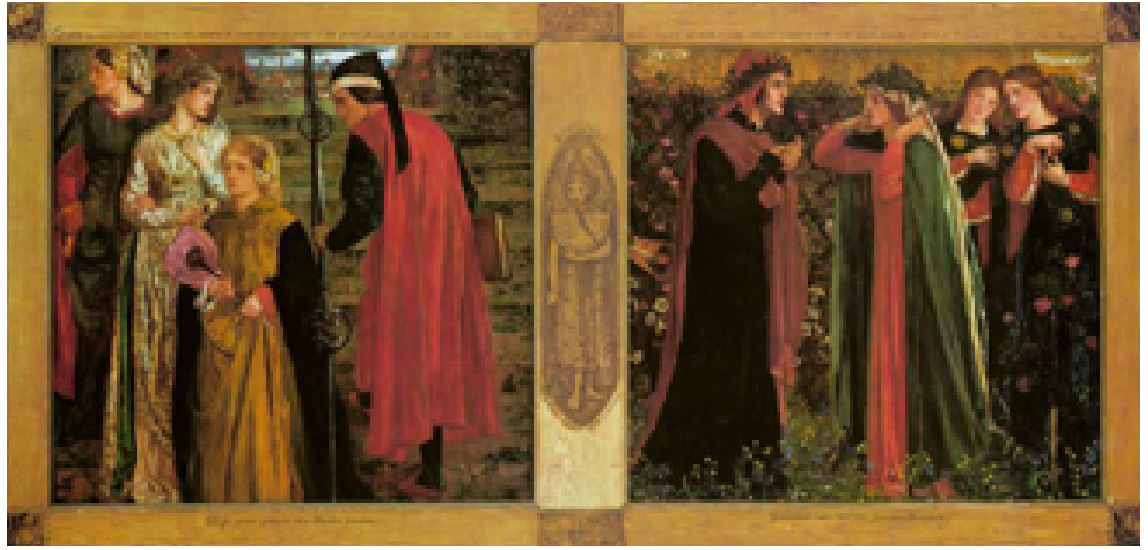

Fig. 2bis: D. G. Rossetti, Salutatio Beatricis, National Gallery of Canada, Ottawa. 


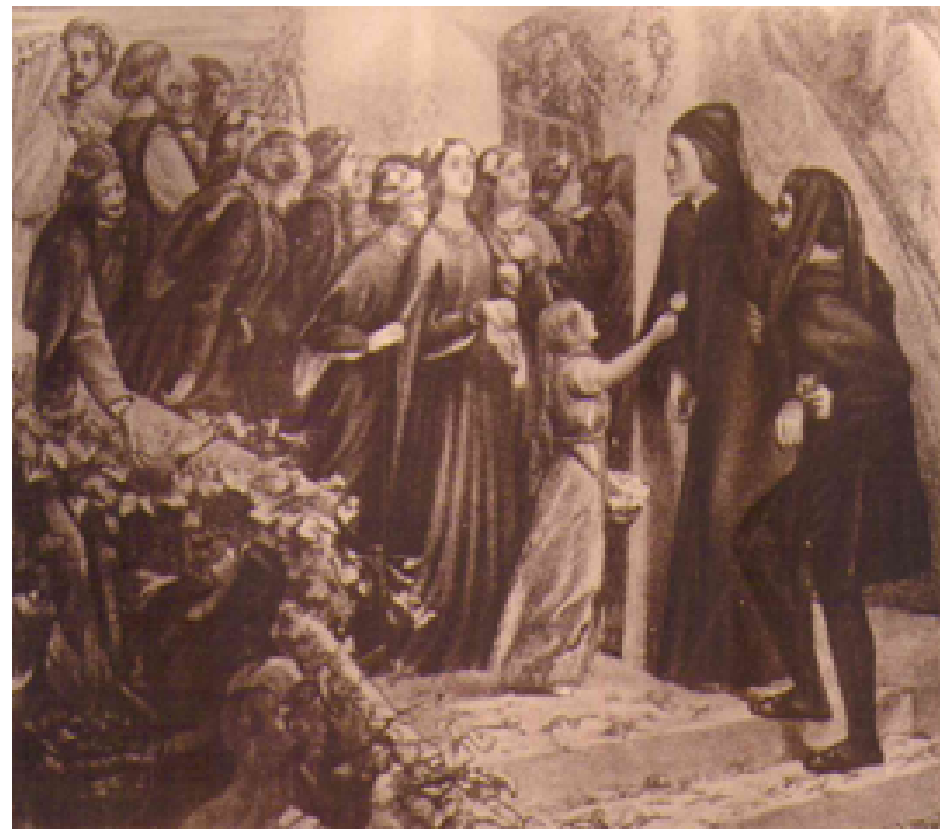

Fig. 3: D. Alighieri, Vita Nuova illustrata dai quadri di Dante Gabriel Rossetti, Roux e Viarengo, Torino, I9O2.

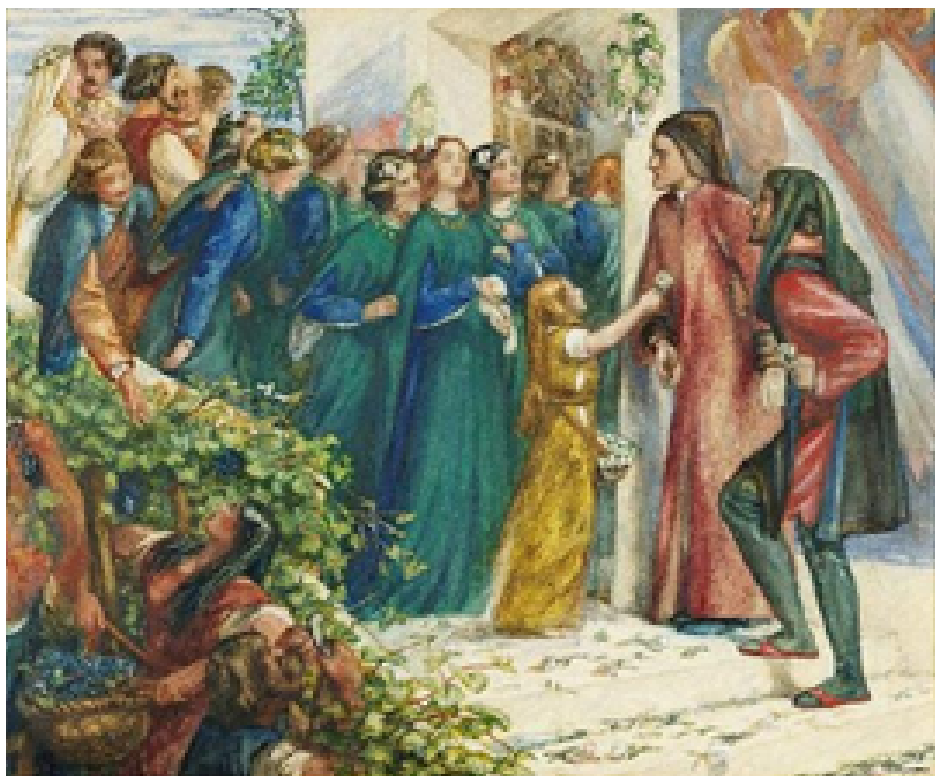

Fig. 3bis: D. G. Rossetti, Beatrice meeting Dante at a Marriage Feast, Denies him her Salutation, collezione privata. 


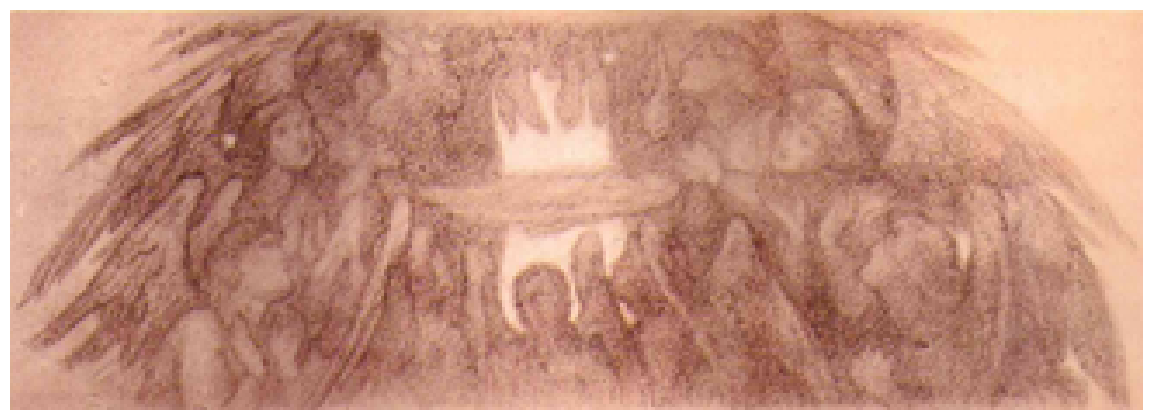

Fig. 4: D. Alighieri, Vita Nuova illustrata dai quadri di Dante Gabriel Rossetti, Roux e Viarengo, Torino, 1902.

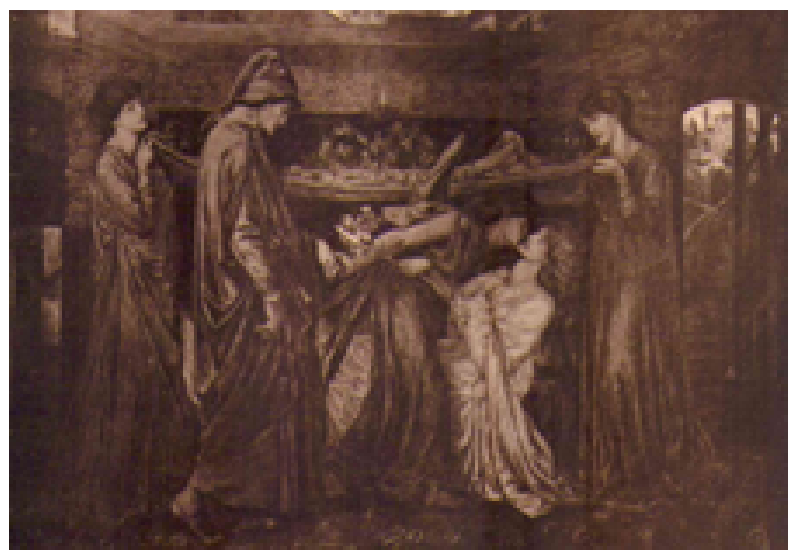

Fig. 5: D. Alighieri, Vita Nuova illustrata dai quadri di Dante Gabriel Rossetti, Roux e Viarengo, Torino, 1902.

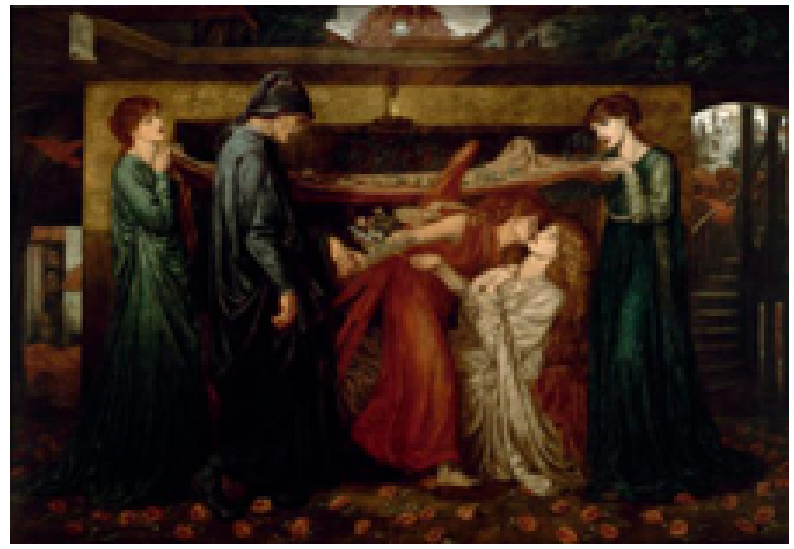

Fig. sbis: D. G. Rossetti, Dante’s Dream, Walker Art Gallery, Liverpool. 

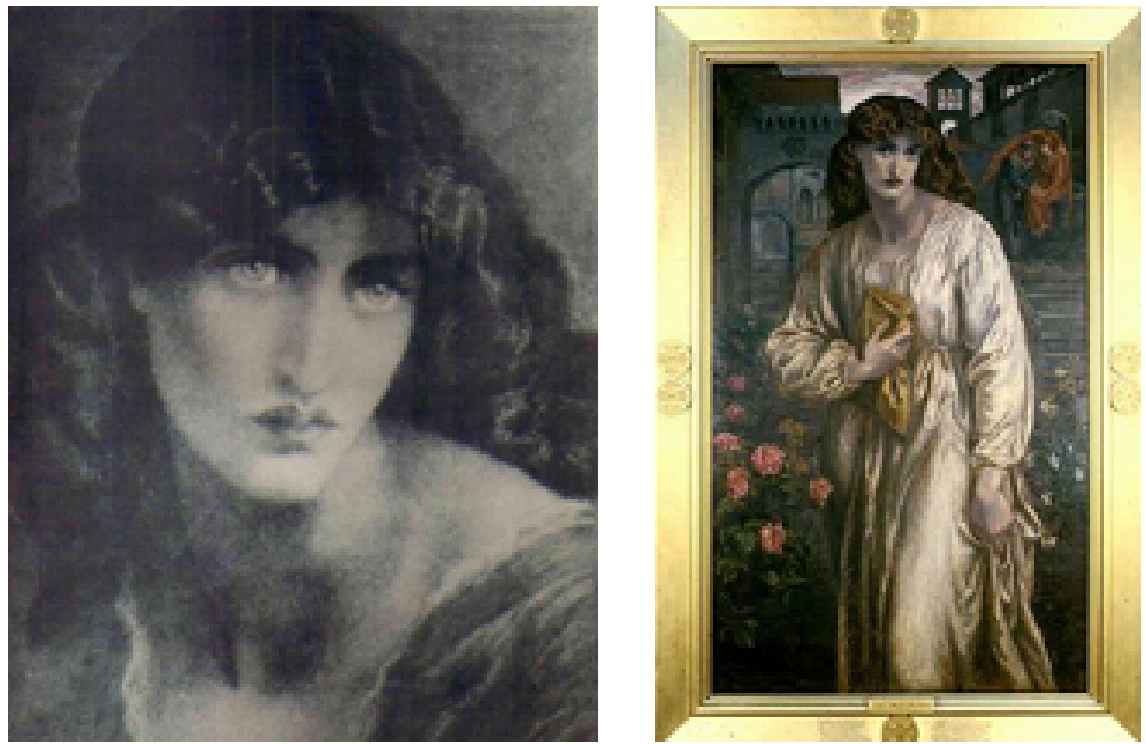

Fig. 6: D. Alighieri, Vita Nuova illustrata dai quadri di Dante Gabriel Rossetti, Roux e Viarengo, Torino, 1902.

Fig. 6 bis: D. G. Rossetti, The salutation of Beatrice, Museum of Art, Toledo (Ohio).

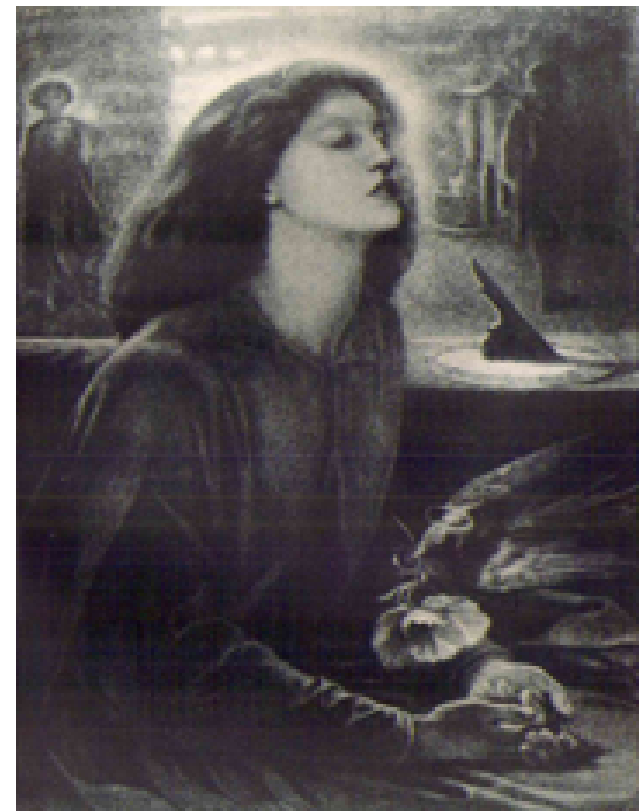

Fig. 7: D. Alighieri, Vita Nuova illustrata dai quadri di Dante Gabriel Rossetti, Roux e Viarengo, Torino, 1902. 


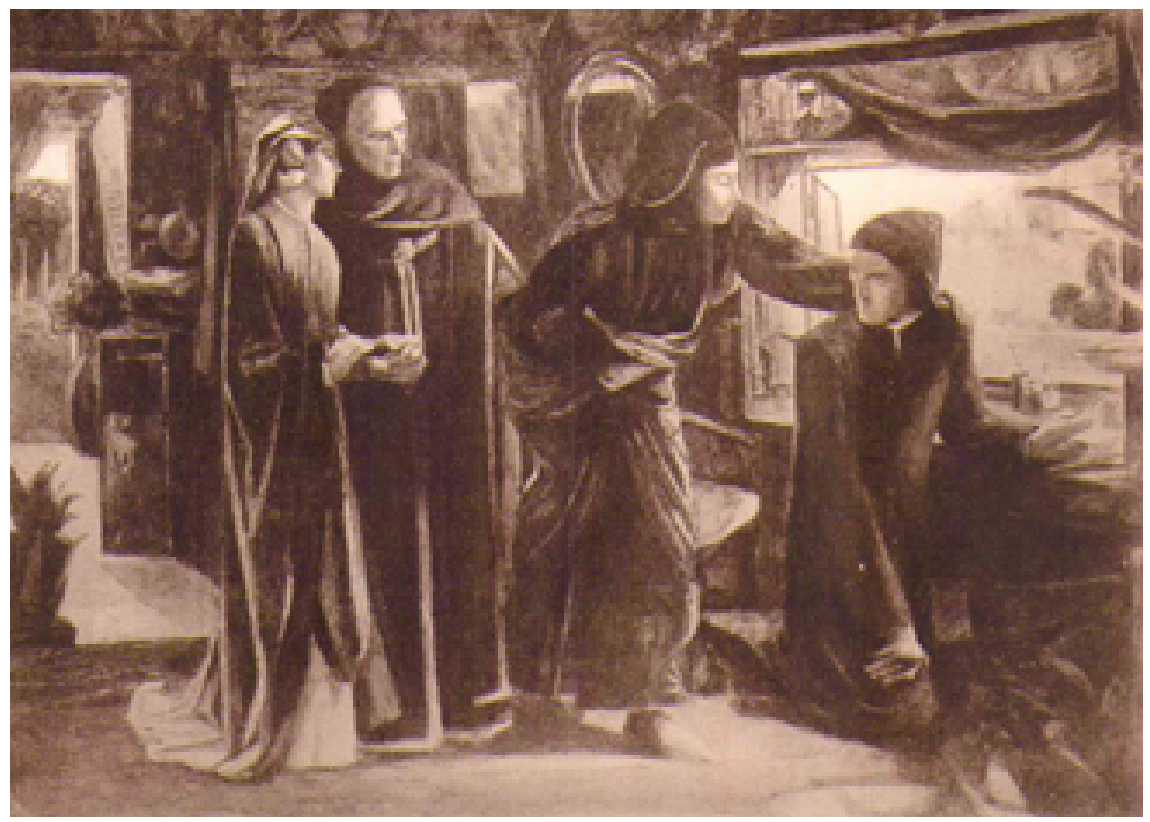

Fig. 8: D. Alighieri, Vita Nuova illustrata dai quadri di Dante Gabriel Rossetti, Roux e Viarengo, Torino, 1902.

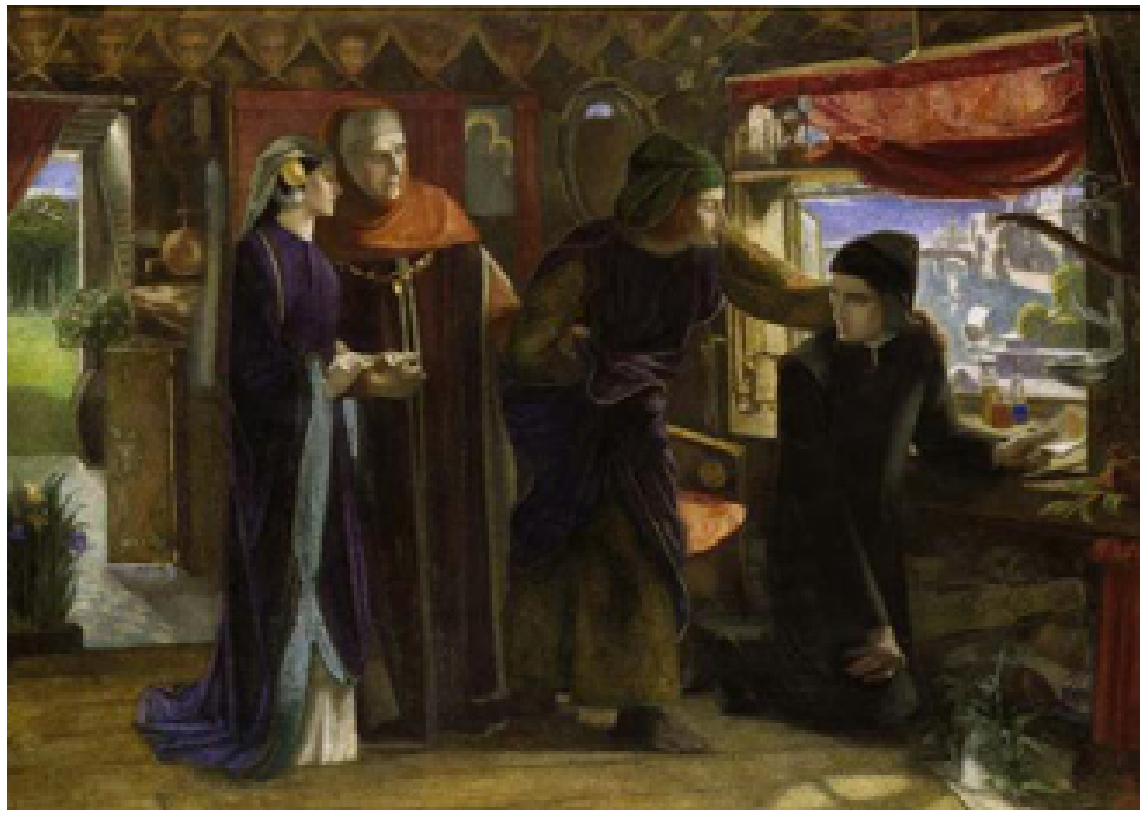

Fig. 8bis: D. G. Rossetti, The First Anniversary of the Death of Beatrice, Ashmolean Museum, Oxford. 


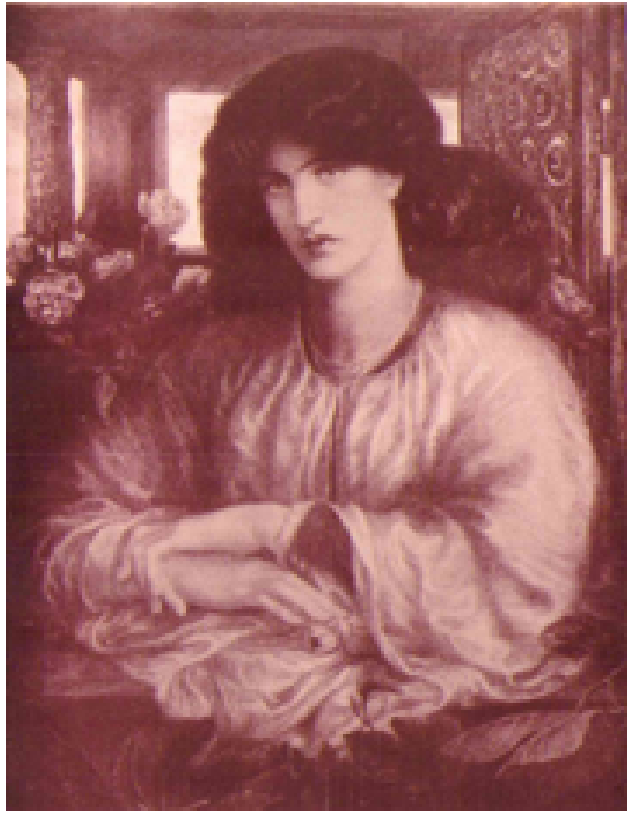

Fig. 9: D. Alighieri, Vita Nuova illustrata dai quadri di Dante Gabriel Rossetti, Roux e Viarengo, Torino, 1902.

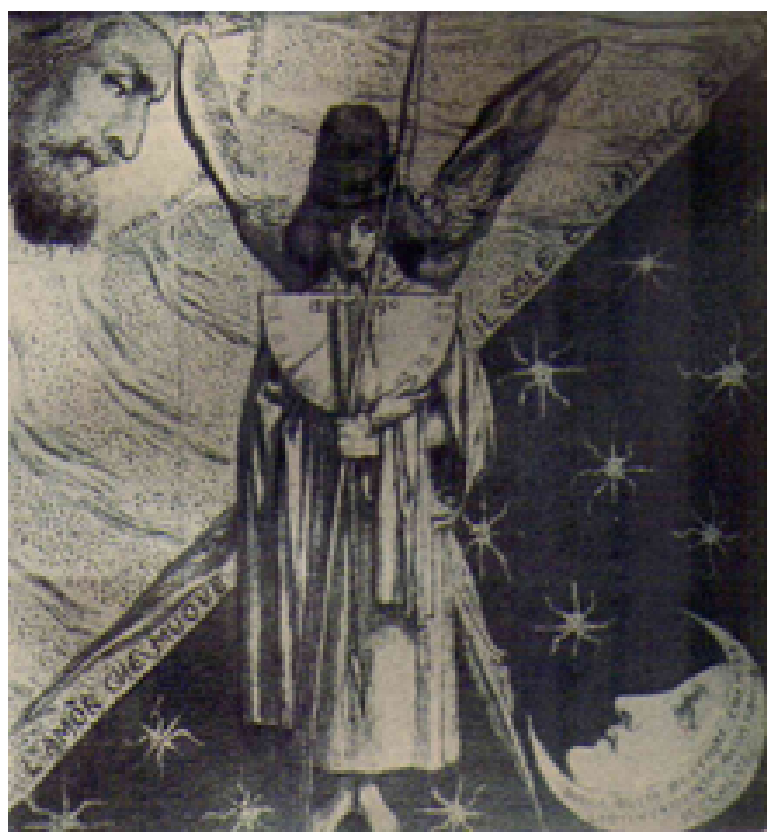

Fig.Io: D. Alighieri, Vita Nuova illustrata dai quadri di Dante Gabriel Rossetti, Roux e Viarengo, Torino, 1902. 


\section{BiBLIOGRAFIA}

Barringer, T., Beatrice, L., Prettejohn, E., Rosenfeld, J., Smith A., 20I4, Preraffaelliti. L'utopia della bellezza, Catalogo della mostra tenuta a Palazzo Chiablese, Torino, I8 aprile - I3 luglio 20I4, Milano, 24 ore cultura.

Barbi, M., I932 (a cura di), Dante Alighieri, La Vita Nuova, Firenze, Bemporad.

Becker, E., Prettejohn, E., Treuherz, J., 2004, Dante Gabriel Rossetti [Catalogo della Mostra tenuta a Liverpool e Amsterdam nel 2003-2004], Liverpool, Waanders.

Benedetti, M. T, 1998, Dante Gabriel Rossetti, Milano, Charta.

Bertolucci, V., 1989, Morfologie del testo medievale, Bologna, il Mulino.

Bonetti, G., 20II, Dante Gabriel Rossetti, Edward Burne-Jones e il mito dell'Italia nell'Inghilterra vittoriana, a cura di M. T. Benedetti, S. Frezzotti, R. Upstone, Ministero per i Beni e le Attività Culturali, Milano, Electa, pp. I76-I83.

Camilletti, F., 2003, "The golden veil. Purezza e malinconia in un racconto di Dante Gabriel Rossetti", Rivista di Studi Vittoriani, VIII, I5, pp. 77-93.

-, 2005, Beatrice nell'inferno di Londra: saggio su Dante Gabriel Rossetti, Lavis, La Finestra.

Caputo, R., 1993, Per far segno: la critica dantesca americana da Singleton a oggi, Roma, Il calamo.

Carù, Carlo (a cura di), 2013, Idee su Dante. Esperimenti danteschi. Atti del Convegno (Milano 9-Io maggio 20I2), Firenze, Società Editrice Fiorentina.

Ceccarelli, S., 20II, Dante G. Rossetti, interprete e traduttore della lirica italiana antica, tesi di dottorato, relatore P. Canettieri, Università di Roma "La Sapienza”. [http:// padis.uniromar.it/handle/Io805/I770]

Ciccuto, M., 1994, “'Era venuta ne la mente mia' ( $V . N$., XXXIV, 7): la visione nel libello e l'immagine in Dante", La gloriosa donna de la mente. A commentary on the "Vita Nuova", a cura di V. Moleta, Firenze, Olschki, pp. I8I-I93.

Circeo, E., 1984, "Per un'analisi della 'Beatrice di Dante' di Gabriele Rossetti”, I Rossetti tra Italia e Inghilterra. Atti del Convegno internazionale di studi (Vasto, 2324-25 settembre I982), a cura di G. Oliva, Roma, Bulzoni, pp. I9I-I94.

De Robertis, D. (a cura di), I980, Dante Alighieri, Vita Nuova, Milano-Napoli, Ricciardi.

Evangelista, S., 20I4, "Echi intertestuali nel Dante a Verona di D.G. Rossetti a partire dalle fonti biografiche dantesche", Sinestesieonline, III, 8. [http://www.rivistasinestesie.it/giugno-20I4.html]

Feo, M., I975, "Pallida no, ma più che neve bianca", Giornale Storico della Letteratura Italiana, a. XCII, vol. CLII, fasc. 479, pp. 32I-36I.

Fredeman, W. E., 2002-20Io, The Correspondence of Dante Gabriel Rossetti, IX voll., Cambridge, Brewer.

Gizzi, C. (a cura di), 1985, Dante Alighieri, Vita nuova, con traduzione inglese e illustrazioni di D. G. Rossetti, Milano, Mazzotta.

—, I999, Dante istoriato. Vent'anni di ricerca iconografica dantesca, Milano, Skira.

Gorni, G. (a cura di), I996, Dante Alighieri, Vita Nova, Torino, Einaudi.

Hinterhäuser, H., I994, "La figura di Beatrice all'insegna del Romanticismo e del Simbolismo", Beatrice nell'opera di Dante e nella memoria europea (I290-I990). Atti del Convegno Internazionale (I0-I4 dicembre 199o), a cura di M. Picchio Simonelli, Firenze, Cadmo, pp. 38I-398.

Marini, M. F., 20II, Dante Gabriel Rossetti, Edward Burne-Jones e il mito dell'Italia nell'Inghilterra vittoriana, a cura di M. T. Benedetti, S. Frezzotti, R. Upstone, 
Ministero per i Beni e le Attività Culturali, Milano, Electa, pp. I84-I96.

Miralles, L., I989, "Dantis Amor: Gabriele Rossetti e il 'paradigma del velame”, L'idea deforme. Interpretazioni esoteriche di Dante, a cura di M. P. Pozzato, intr. di U. Eco, postf. di A. A. Rosa, Milano, Bompiani, pp. 47-77.

Pisanti, T., 1984, "I 'primitivi' italiani e la poesia di D.G. Rossetti", I Rossetti tra Italia e Inghilterra. Atti del Convegno internazionale di studi (Vasto, 23-24-25 settembre I982), a cura di G. Oliva, Bulzoni, Roma, pp. 239-250.

Renzi, L., 2007, Le conseguenze di un bacio, Bologna, il Mulino.

Rossetti, W. M., I889, Dante Gabriel Rossetti as designer and writer, London, Cassel.

Santagata, M., 20II, L'io e il mondo. Un'interpretazione di Dante, Bologna, il Mulino.

Surtees, V., I97I, The paintings and drawings of Dante Gabriele Rossetti (I828-I882). A Catalogue Raisonné, Oxford, Clarendon [il numero, con l'eventuale lettera, fa riferimento al vol. I e indica la scheda di catalogo; l'indicazione "pl." vale "plate" e fa riferimento alla numerazione delle tavole nel vol. II]. 